\title{
Observing Iron Under Pressure
}

\author{
Femtosecond-resolved $x$-ray diffraction images of iron's crystals as they \\ deform under an extreme load show that the material's elastic-plastic \\ transition comes after a surprisingly long elastic phase.
}

\section{By Marric Stephens}

N nowing how materials respond to extreme loads is vital to understanding phenomena from debris impacts in jet engines to planetary formation. But experimental challenges in capturing material responses mean empirical data are patchy; instead, material behaviors must generally be predicted theoretically. Now, Sébastien Merkel at the University of Lille, France, and colleagues have provided much-needed ground truth for theory by observing directly how the crystal structure of iron evolves as it deforms at high strain rates [1].

The team fixed a 50- $\mu$ m-thick polymer film to a $25-\mu \mathrm{m}$-thick iron foil and blasted the polymer with a 12-ns laser pulse to send a shock wave into the iron. For each sample, the researchers used femtosecond $\mathrm{x}$-ray diffraction to capture a single snapshot of the structure and orientation of the iron's crystals and the stress imparted by the shock wave. By varying the pulse-measurement interval over a series of experiments, they built a time-resolved record of how the stress developed and the iron crystals deformed.
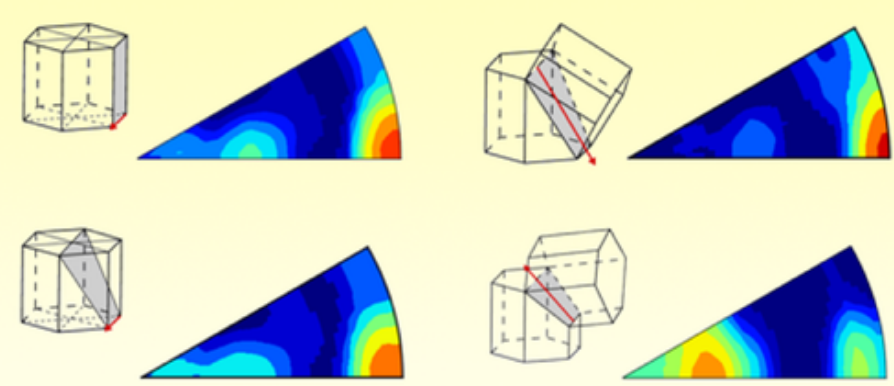

Initially, the shock wave changed the iron's structure from body-centered-cubic to hexagonal-close-packed, something the team expected to happen. The hexagonal structure then deformed elastically for several nanoseconds before yielding, after which it accommodated strain by rearranging itself into pairs of twinned crystals-a process that continued even after the stress had fallen below the yield stress. Both the time to yielding and the mechanism were previously unknown. Merkel and his colleagues attribute the observed "elastic overshoot" to the relatively slow buildup of twinning nuclei during the elastic phase: only when enough had accumulated could deformation via twinning begin.

Marric Stephens is a Corresponding Editor for Physics based in Bristol, UK.

\section{REFERENCES}

1. S. Merkel et al., "Femtosecond visualization of hcp-iron strength and plasticity under shock compression,” Phys. Rev. Lett. 127, 205501 (2021).

Credit: S. Merkel et al. [1] 